\title{
Efficacy and Safety of Retinol Palmitate Ophthalmic Solution in the Treatment of Dry Eye: A Japanese Phase II Clinical Trial [Corrigendum]
}

Toshida H, Funaki T, Ono K, et al. Drug Des Devel Ther. 2017;11:1871-1879

The authors have advised there are some mistakes in the mean changes and standard errors of the paper:

1. The standard errors of groups Placebo and VApal were all the same value for staining and symptom scores in Figure 1 (on page 1875) and Figure 3 (on page 1876), and Table 3 (on page 1877).

2. There were slight discrepancies in the Mean changes of Placebo group at 1, 2 and 4 weeks in Figure 3D.

3. There was a slight discrepancy in the Mean change of foreign body sensation in Placebo group at 1 week in Table 3.

The authors have advised the errors were due to:
1. The standard error listed in a different table on the statistical analysis software was adopted.

2. There were typing errors when transferring numerical values from statistical analysis software to graph creation software.

3. There was a mistake when rounding numerical value.

Since the statistical analysis had been performed using the original mean values (correct values), all results do not change.

The authors apologize for these errors and advise they do not affect the results of the paper.

The updated Figures and Table are shown below.
A

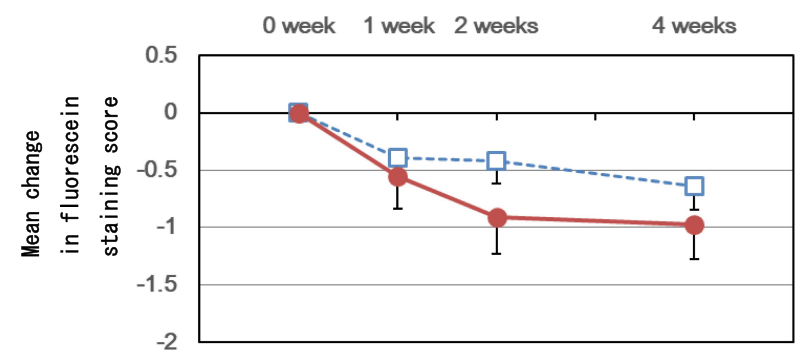

B

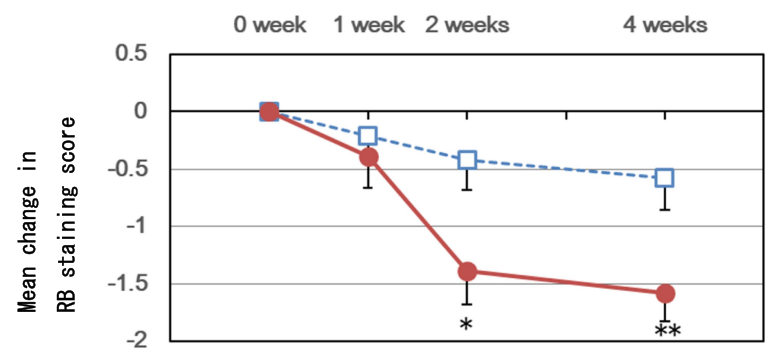

-- --- Placebo - VApal

Figure I Mean change in staining score.

Notes: Mean change in fluorescein staining score $(\mathbf{A})$ and RB staining score (B) from baseline to I, 2 , and 4 weeks. $* \mathrm{P}<0.05$. $* * \mathrm{P}<0.0 \mathrm{I}$ versus placebo (unpaired $t$-test). Abbreviations: RB, rose bengal; VApal, retinol palmitate. 

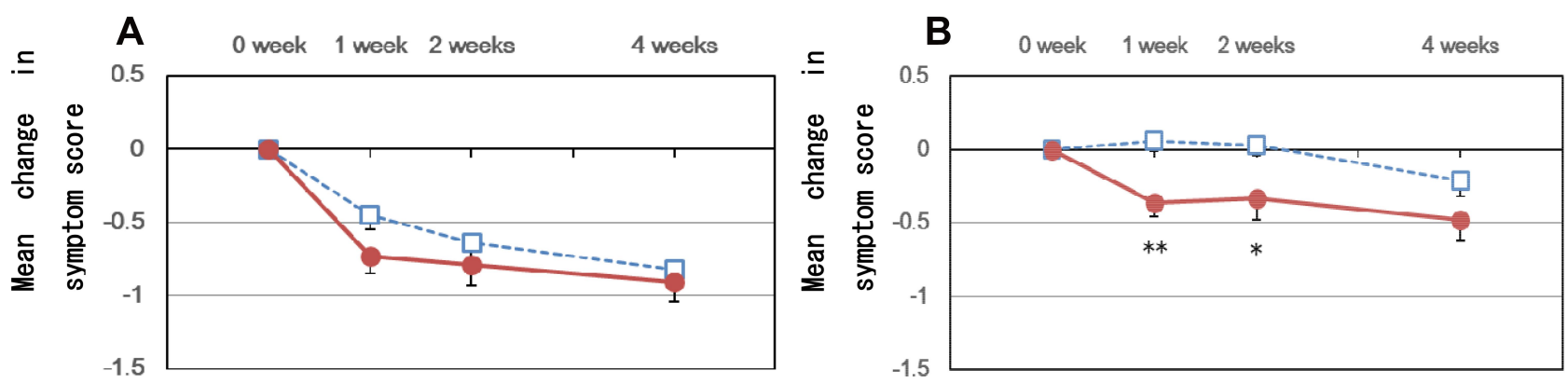

C
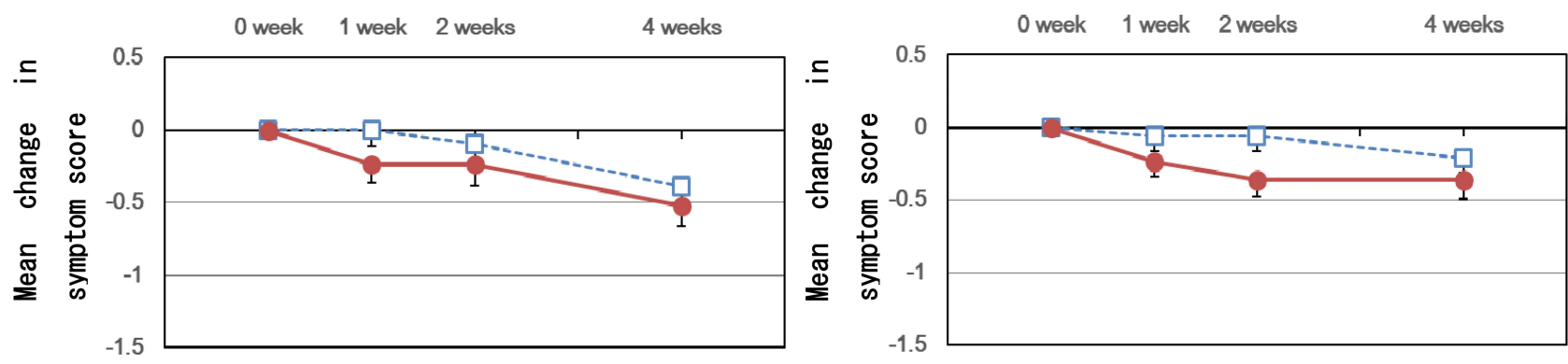

$\mathbf{E}$

$\mathbf{F}$
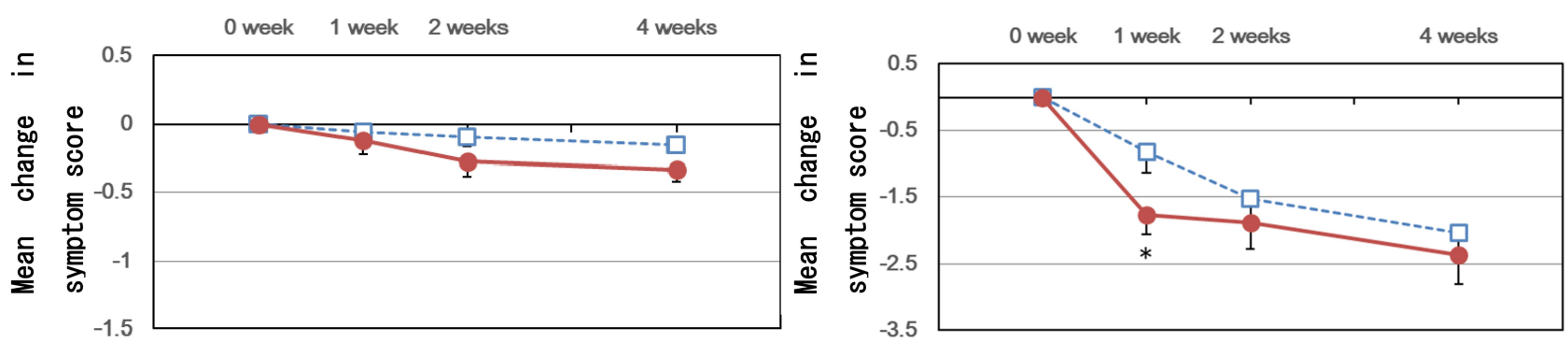

--口-- Placebo - VApal

Figure 3 Mean change in symptom score.

Notes: Mean change in symptom scores for dryness (A), blurred vision (B), heaviness (C), itching (D), and eye discharge (E) and total score for subjective symptoms (F) from baseline to I, 2, and 4 weeks. ${ }^{*} \mathrm{P}<0.05$. ${ }^{*} * \mathrm{P}<0.0 \mathrm{I}$ versus placebo (unpaired $t$-test).

Abbreviation: VApal, retinol palmitate. 
Table 3 Mean Change in Symptom Score

\begin{tabular}{|c|c|c|c|c|}
\hline & \multirow[t]{2}{*}{ Week } & \multicolumn{2}{|c|}{ Mean Change } & \multirow[t]{2}{*}{$P$-value } \\
\hline & & Placebo & VApal & \\
\hline \multirow[t]{3}{*}{ Dryness } & I & $-0.45 \pm 0.10$ & $-0.73 \pm 0.12$ & 0.0794 \\
\hline & 2 & $-0.64 \pm 0.11$ & $-0.79 \pm 0.14$ & 0.3957 \\
\hline & 4 & $-0.82 \pm 0.13$ & $-0.91 \pm 0.13$ & 0.6224 \\
\hline \multirow[t]{3}{*}{ Eye fatigue } & 1 & $-0.33 \pm 0.12$ & $-0.30 \pm 0.10$ & 0.8483 \\
\hline & 2 & $-0.48 \pm 0.12$ & $-0.39 \pm 0.12$ & 0.5923 \\
\hline & 4 & $-0.55 \pm 0.11$ & $-0.58 \pm 0.14$ & 0.8629 \\
\hline \multirow[t]{3}{*}{ Foreign body sensation } & I & $-0.09 \pm 0.13$ & $-0.30 \pm 0.14$ & 0.2780 \\
\hline & 2 & $-0.36 \pm 0.13$ & $-0.33 \pm 0.13$ & 0.8683 \\
\hline & 4 & $-0.33 \pm 0.14$ & $-0.42 \pm 0.16$ & 0.6693 \\
\hline \multirow[t]{3}{*}{ Blurred vision } & I & $0.06 \pm 0.07$ & $-0.36 \pm 0.10$ & 0.0016 \\
\hline & 2 & $0.03 \pm 0.07$ & $-0.33 \pm 0.15$ & 0.0299 \\
\hline & 4 & $-0.2 I \pm 0.11$ & $-0.48 \pm 0.14$ & 0.1321 \\
\hline \multirow[t]{3}{*}{ Eye pain } & I & $-0.27 \pm 0.16$ & $-0.21 \pm 0.09$ & 0.7506 \\
\hline & 2 & $-0.45 \pm 0.16$ & $-0.24 \pm 0.12$ & 0.2813 \\
\hline & 4 & $-0.48 \pm 0.17$ & $-0.33 \pm 0.16$ & 0.5258 \\
\hline \multirow[t]{3}{*}{ Photophobia } & 1 & $-0.06 \pm 0.10$ & $-0.15 \pm 0.08$ & 0.4646 \\
\hline & 2 & $-0.09 \pm 0.09$ & $-0.18 \pm 0.11$ & 0.5276 \\
\hline & 4 & $-0.18 \pm 0.12$ & $-0.21 \pm 0.08$ & 0.8359 \\
\hline \multirow[t]{3}{*}{ Heaviness } & I & $0.00 \pm 0.11$ & $-0.24 \pm 0.12$ & 0.1277 \\
\hline & 2 & $-0.09 \pm 0.12$ & $-0.24 \pm 0.14$ & 0.4199 \\
\hline & 4 & $-0.39 \pm 0.11$ & $-0.52 \pm 0.14$ & 0.5025 \\
\hline \multirow[t]{3}{*}{ Itching } & I & $-0.06 \pm 0.10$ & $-0.24 \pm 0.10$ & 0.1905 \\
\hline & 2 & $-0.06 \pm 0.10$ & $-0.36 \pm 0.12$ & 0.0556 \\
\hline & 4 & $-0.21 \pm 0.10$ & $-0.36 \pm 0.13$ & 0.3652 \\
\hline \multirow[t]{3}{*}{ Ocular discomfort } & 1 & $-0.27 \pm 0.14$ & $-0.24 \pm 0.14$ & 0.8806 \\
\hline & 2 & $-0.33 \pm 0.10$ & $-0.27 \pm 0.14$ & 0.7284 \\
\hline & 4 & $-0.42 \pm 0.14$ & $-0.55 \pm 0.13$ & 0.5369 \\
\hline \multirow[t]{3}{*}{ Eye discharge } & I & $-0.06 \pm 0.04$ & $-0.12 \pm 0.10$ & 0.5923 \\
\hline & 2 & $-0.09 \pm 0.07$ & $-0.27 \pm 0.11$ & 0.1600 \\
\hline & 4 & $-0.15 \pm 0.06$ & $-0.33 \pm 0.09$ & 0.1137 \\
\hline \multirow[t]{3}{*}{ Tearing } & $\mathrm{I}$ & $-0.06 \pm 0.04$ & $-0.15 \pm 0.06$ & 0.2369 \\
\hline & 2 & $-0.09 \pm 0.07$ & $-0.12 \pm 0.07$ & 0.7593 \\
\hline & 4 & $-0.06 \pm 0.07$ & $-0.12 \pm 0.10$ & 0.6384 \\
\hline \multirow[t]{3}{*}{ Hyperemia } & $\mathrm{I}$ & $0.00 \pm 0.06$ & $-0.06 \pm 0.12$ & 0.6602 \\
\hline & 2 & $-0.06 \pm 0.10$ & $-0.03 \pm 0.13$ & 0.8551 \\
\hline & 4 & $-0.21 \pm 0.10$ & $-0.12 \pm 0.12$ & 0.5719 \\
\hline \multirow[t]{3}{*}{ Total score of the subjective symptoms } & 1 & $-0.82 \pm 0.32$ & $-1.76 \pm 0.30$ & 0.0351 \\
\hline & 2 & $-1.52 \pm 0.31$ & $-1.88 \pm 0.39$ & 0.4695 \\
\hline & 4 & $-2.03 \pm 0.44$ & $-2.36 \pm 0.45$ & 0.5983 \\
\hline
\end{tabular}

Abbreviation: VApal, retinol palmitate. 


\section{Publish your work in this journal}

Drug Design, Development and Therapy is an international, peerreviewed open-access journal that spans the spectrum of drug design and development through to clinical applications. Clinical outcomes, patient safety, and programs for the development and effective, safe, and sustained use of medicines are a feature of the journal, which has also been accepted for indexing on PubMed Central. The manuscript management system is completely online and includes a very quick and fair peer-review system, which is all easy to use. Visit http://www. dovepress.com/testimonials.php to read real quotes from published authors. 